\title{
Optimal Feedback Interval for Temporally-Correlated Multiantenna Channel
}

\author{
Wiroonsak Santipach and Kritsada Mamat \\ Department of Electrical Engineering \\ Faculty of Engineering, Kasetsart University \\ Bangkok, Thailand 10900 \\ Email: \{wiroonsak.s, g5317500192\}@ku.ac.th
}

\begin{abstract}
Assuming perfect channel state information (CSI), the receiver in a point-to-point multiantenna channel can compute the optimal transmit beamforming vector that maximizes channel capacity. The transmitter, which is not able to estimate the CSI, obtains the quantized transmit beamforming vector via a rate-limited feedback channel. We assume that time evolution of both MIMO and MISO channels can be modeled as the first-order autoregressive process parameterized by a temporalcorrelation coefficient. For a limited number of feedback bits, we would like to find out how often the feedback update should take place. Applying a large system limit and random vector quantization (RVQ), we derive the integer optimization problem, which determines the optimal feedback interval that maximizes the average capacity. The analytical results show that the optimal feedback interval depends on the temporal correlation coefficient, available feedback, and the number of transmit and receive antennas.
\end{abstract}

\section{INTRODUCTION}

Multiple-antenna array has been included in technical specifications of the latest cellular telephonies (e.g., LTE-advanced and WIMAX). Employing multiple antennas at the transmitter and/or receiver has been shown to increase spatial diversity and spectral efficiency [1], [2]. For full potential of the multipleantenna system, channel state information (CSI) at both the transmitter and receiver is required. At the receiver, CSI can be estimated from pilot signals and the accuracy of CSI estimate greatly affects the system performance [3], [4].

In a time-division duplex (TDD) with channel reciprocity, the transmitter is able to estimate the channel from pilots sent by the receiver. However, estimating channel at the transmitter is not possible in a frequency-division duplex (FDD) where forward and backward channels are in different frequency bands. Consequently, the transmitter in FDD must obtain CSI from the receiver via a low-rate feedback channel. Recently, many researchers have proposed feedback schemes to quantize CSI and analyzed associated performance (see [5] and references therein). In our work, we assume that the receiver has perfect CSI and thus, can compute the optimal transmit beamforming vector that maximizes an instantaneous channel capacity. With limited feedback, the beamforming vector is selected from a quantization set or a codebook, which is known

This work was supported by Kasetsart University Research and Development Institute (KURDI) under the FY 2010 KU research grant and the 2010 Telecommunications Research and Industrial Development Institute (TRIDI) scholarship. a priori at the transmitter and the receiver. The codebook index of the selected vector is then fed back to the transmitter, which subsequently adjusts its beamforming coefficients. Different codebooks have been proposed and analyzed in [5]-[8].

Feeding back quantized beamforming coefficients may not be useful in a fast fading channel since they can be quickly outdated. If the channel fades slowly, the beamforming coefficients may not need to be updated frequently. Thus, a feedback strategy should be adapted to a temporal correlation of the channel and was considered in [9]-[12]. Switched codebook quantization was proposed in [9] where the codebook selection is based on channel spatial and temporal correlations. In [10], quantized CSI is modeled as the first-order finite-state Markov chain and the beamforming feedback is based on channel dynamics. In [11], [12], multiple-input and single-output (MISO) channel is modeled as a first-order autoregressive process and the frequency of feedback updates is determined by temporal correlation of the channel.

Here we consider block fading for both MISO and multipleinput multiple-output (MIMO) channels with time evolution modeled by the first-order autoregressive process. We apply random vector quantization (RVQ) codebook to quantize the transmit beamformer. The RVQ codebook consists of independent isotropically distributed vectors and is shown to perform close to the optimum codebook [6], [13]. Furthermore, RVQ can be analyzed to obtain some insights into the limited feedback performance. We derive exact expressions for the average capacity of both MISO and MIMO channels in a large system limit in which the number of transmit and receive antennas and feedback bits tend to infinity with fixed ratios. For a given feedback budget, we maximize the large system capacity over a feedback interval. We show that the solution is a function of temporal correlation, the number of feedback bits, and the ratio between number of transmit and receive antennas and that selecting the right feedback interval can increase the performance significantly.

\section{Channel Model}

We consider a point-to-point discrete-time multiantenna channel with $N_{t}$ transmit and $N_{r}$ receive antennas. We assume a block fading in which channel gains remain static for the whole coherence period and change at the next coherence period. To allow a meaningful feedback of CSI from the 
receiver, the coherence period is assumed to be sufficiently long. During the $k$ th fading block, an $N_{r} \times 1$ receive vector is given by

$$
\boldsymbol{r}=\boldsymbol{H}(k) \boldsymbol{v}(k) x_{s}+\boldsymbol{n}
$$

where $x_{s}$ is a transmitted symbol with zero mean and unit variance, $\boldsymbol{n}$ is an $N_{r} \times 1$ AWGN vector with zero mean and covariance $\sigma_{n}^{2} \boldsymbol{I}, \boldsymbol{v}(k)$ is an $N_{t} \times 1$ unit-norm beamforming vector for the $k$ th fading block, and $\boldsymbol{H}(k)=\left[h_{i j}(k)\right]$ is an $N_{r} \times N_{t}$ channel matrix whose element $h_{i j}(k)$ is the channel gain between the $i$ th transmit and the $j$ th receive antennas during the $k$ th fading block. Here we consider a rankone transmit precoding or beamforming. An arbitrary-rank transmit precoding with multiple independent data streams was considered in [6]. Assuming an ideal scattering environment, $h_{i j}(k)$ is modeled as a complex Gaussian random variable with zero mean and unit variance. Also we assume that adjacent antennas in antenna arrays at both the transmitter and receiver are placed sufficiently far apart that elements of $\boldsymbol{H}(k)$ are independent.

To model a time evolution of the channel considered, we adopt the first-order autoregressive process, which has been widely used and was shown to predict channel dynamics well [9], [11], [14], [15]. Thus, the channel matrix of the $k$ th fading block relates to that of the previous block as follows

$$
\boldsymbol{H}(k)=\alpha \boldsymbol{H}(k-1)+\sqrt{1-\alpha^{2}} \boldsymbol{W}(k)
$$

where $\boldsymbol{H}(k-1)$ is the channel matrix for the $k-1$ th fading block, $\boldsymbol{W}(k)$ is an $N_{r} \times N_{t}$ matrix with independent zeromean unit-variance complex Gaussian entries, and $\alpha \in[0,1]$ denotes a temporal correlation coefficient between adjacent blocks. Note that $\alpha=1$ produces a time-invariant channel. On the other hand, $\alpha=0$ indicates a channel with no temporal correlation and thus, the channel fades independently from one coherence block to the next.

The associated ergodic capacity of this channel is given by

$$
C=E \log \left(1+\rho \boldsymbol{v}^{\dagger}(k) \boldsymbol{H}^{\dagger}(k) \boldsymbol{H}(k) \boldsymbol{v}(k)\right)
$$

where $\rho=1 / \sigma_{n}^{2}$ denotes the background signal-to-noise ratio (SNR) and the expectation is over the channel matrix. To achieve the capacity, transmitter encodes the transmitted symbols across many different fading blocks. In addition to SNR, the capacity also depends on the beamforming vector $\boldsymbol{v}(k)$. If the transmitter can track the channel completely (perfect CSI), the optimal $\boldsymbol{v}$ is the eigenvector of the channel covariance $\boldsymbol{H}^{\dagger}(k) \boldsymbol{H}(k)$ corresponding to the maximum eigenvalue. In other words, the optimal beamforming vector is in the direction of the strongest channel mode.

In a FDD system, the transmitter is not able estimate the channel directly and has to rely on CSI fed back from the receiver via a rate-limited channel. The receiver can estimate channel from pilot signals, which is known a priori at the transmitter and receiver. Assuming perfect CSI, the receiver selects the optimal beamforming vector and send it back via the feedback channel to the transmitter. Since the feedback channel is rate-limited, the selected beamforming vector needs to be quantized. Here we quantize the transmit beamforming vector with random vector quantization (RVQ) codebook

$$
\mathcal{V}=\left\{\boldsymbol{v}_{1}, \boldsymbol{v}_{2}, \cdots, \boldsymbol{v}_{n}\right\}
$$

whose entries $\boldsymbol{v}_{j}$ are independent isotropically distributed and $n$ denotes the number of entries in the RVQ codebook. For given $B=\log _{2} n$ feedback bits for quantization, RVQ performs close to the optimal codebook [6], [13] and RVQ is optimal (i.e. maximizing capacity) in a large system limit [6], [7]. The large system limit refers to the limit in which $N_{t}, N_{r}, B$ tend to infinity with fixed $\bar{N}_{r} \triangleq N_{r} / N_{t}$ and $\bar{B} \triangleq B / N_{t}$.

Given $B$ bits and channel matrix $\boldsymbol{H}(k)$, the receiver selects from the RVQ codebook

$$
\begin{aligned}
\hat{\boldsymbol{v}}(k) & =\arg \max _{\boldsymbol{v}_{j} \in \mathcal{V}} \log \left(1+\rho \boldsymbol{v}_{j}^{\dagger} \boldsymbol{H}^{\dagger}(k) \boldsymbol{H}(k) \boldsymbol{v}_{j}\right) \\
& =\arg \max _{\boldsymbol{v}_{j} \in \mathcal{V}} \boldsymbol{v}_{j}^{\dagger} \boldsymbol{H}^{\dagger}(k) \boldsymbol{H}(k) \boldsymbol{v}_{j} .
\end{aligned}
$$

The index of the selected beamforming vector is then fed back to the transmitter, which adjusts its beamforming vector accordingly. We assume that a time duration to feed back the selected index is negligible when compared to one fading block and that the feedback channel is error-free. The associated capacity with quantized transmit beamformer is given by

$$
C=E \log \left(1+\rho \hat{\boldsymbol{v}}^{\dagger}(k) \boldsymbol{H}^{\dagger}(k) \boldsymbol{H}(k) \hat{\boldsymbol{v}}(k)\right) .
$$

With infinite feedback $(\bar{B} \rightarrow \infty)$ and fixed $\bar{N}_{r}$, the capacity increases with $\log \left(\rho N_{t}\right)$ [6]. Thus, for finite feedback, there is capacity loss denoted by

$$
\begin{aligned}
C_{\triangle} & \triangleq C-\log \left(\rho N_{t}\right) \\
& =E \log \left(\frac{1}{\rho N_{t}}+\frac{1}{N_{t}} \hat{\boldsymbol{v}}^{\dagger}(k) \boldsymbol{H}^{\dagger}(k) \boldsymbol{H}(k) \hat{\boldsymbol{v}}(k)\right) .
\end{aligned}
$$

For an uncorrelated channel $(\alpha=0)$, as $\left(N_{t}, N_{r}, B\right) \rightarrow \infty$,

$$
C_{\triangle} \rightarrow C_{\triangle}^{\infty}=\log \gamma_{\mathrm{rvq}}^{\infty}(\bar{B})
$$

where

$$
\gamma_{\text {rvq }}^{\infty}(\bar{B})=\lim _{\left(N_{t}, N_{r}, B\right) \rightarrow \infty} \frac{1}{N_{t}} \hat{\boldsymbol{v}}^{\dagger}(k) \boldsymbol{H}^{\dagger}(k) \boldsymbol{H}(k) \hat{\boldsymbol{v}}(k)
$$

and the expression for $\gamma_{\mathrm{rvq}}^{\infty}(x)$ is shown in [6], [7] as follows. Suppose

$$
\beta=\frac{1}{\log (2)}\left(\bar{N}_{r} \log \left(\frac{\sqrt{\bar{N}_{r}}}{1+\sqrt{\bar{N}_{r}}}\right)+\sqrt{\bar{N}_{r}}\right) .
$$

For $0 \leq x \leq \beta, \gamma_{\text {rvq }}^{\infty}$ satisfies

$$
\left(\gamma_{\text {rvq }}^{\infty}\right)^{\bar{N}_{r}} \mathrm{e}^{-\gamma_{\text {rvq }}^{\infty}}=2^{-x}\left(\frac{\bar{N}_{r}}{\mathrm{e}}\right)^{\bar{N}_{r}}
$$

and for $x \geq \beta$,

$$
\begin{aligned}
& \gamma_{\mathrm{rvq}}^{\infty}(x)=\left(1+\sqrt{\bar{N}_{r}}\right)^{2}-\exp \left\{\frac{1}{2} \bar{N}_{r} \log \left(\bar{N}_{r}\right)\right. \\
& \left.\quad-\left(\bar{N}_{r}-1\right) \log \left(1+\sqrt{\bar{N}_{r}}\right)+\sqrt{\bar{N}_{r}}-x \log (2)\right\} .
\end{aligned}
$$




\section{OPTIMIZING FEEDBACK INTERVAL}

Since channel is time-varying, the quantized beamforming vector needs to be updated and fed back for every fading block. However, frequent feedbacks reduce spectral efficiency and may not be practical. For a given feedback budget, how often should the feedback update take place? Suppose that there are $B$ feedback bits available per $M$ fading blocks and that for every $K$ fading blocks $(K \leq M)$, the receiver quantizes and feeds the quantized transmit beamforming vector back to the transmitter. There are total $L=M / K$ feedback intervals over $M$ blocks and each feedback update is allocated with $B / L$ feedback bits.

For each feedback interval, we propose that the receiver quantizes the transmit beamformer based on the current channel matrix with $B / L$ bits and that the quantized beamformer is used for subsequent blocks until the next interval. The corresponding average channel capacity over $M$ fading blocks is given by

$$
\begin{aligned}
& \bar{C}_{\triangle} \triangleq \frac{1}{M} \sum_{l=1}^{L} \sum_{k=1}^{K} E \log \left(\frac{1}{\rho N_{t}}\right. \\
&\left.+\frac{1}{N_{t}} \hat{\boldsymbol{v}}^{\dagger}(l K+1) \boldsymbol{H}^{\dagger}(l K+k) \boldsymbol{H}(l K+k) \hat{\boldsymbol{v}}(l K+1)\right) .
\end{aligned}
$$

The proposed method may not be optimal (i.e., maximizing the average capacity) for a given amount of feedback. However, it is simple enough to analyze the capacity and obtain some insights into how often the feedback update should be.

\section{Large System With $\bar{N}_{r}>0$}

To analyze the average capacity in (15), we first compute the effective received power by applying the first-order autoregressive channel model in (2) and some algebraic manipulations. In the large system limit, which we will consider next, the effective received power does not depend on the index of feedback interval $l$, which will be omitted as follows.

$$
\begin{gathered}
\frac{1}{N_{t}} \hat{\boldsymbol{v}}^{\dagger}(1) \boldsymbol{H}^{\dagger}(k) \boldsymbol{H}(k) \hat{\boldsymbol{v}}(1)=\frac{\alpha^{2 k-2}}{N_{t}} \hat{\boldsymbol{v}}^{\dagger}(1) \boldsymbol{H}^{\dagger}(1) \boldsymbol{H}(1) \hat{\boldsymbol{v}}(1) \\
+\left(1-\alpha^{2}\right) \sum_{i=0}^{k-2} \alpha^{2 i} \frac{1}{N_{t}}\|\boldsymbol{W}(k-i) \hat{\boldsymbol{v}}(1)\|^{2} \\
+\left(1-\alpha^{2}\right) \sum_{i=0}^{k-2} \sum_{\substack{j=0 \\
j \neq i}}^{k-2} \frac{\alpha^{i} \alpha^{j}}{N_{t}} \hat{\boldsymbol{v}}^{\dagger}(1) \boldsymbol{W}^{\dagger}(k-i) \boldsymbol{W}(j-i) \hat{\boldsymbol{v}}(1) \\
+2 \alpha \sqrt{1-\alpha^{2}} \sum_{i=0}^{k-2} \frac{\alpha^{i}}{N_{t}} \Re\left\{\hat{\boldsymbol{v}}^{\dagger}(1) \boldsymbol{H}^{\dagger}(1) \boldsymbol{W}(k-i) \hat{\boldsymbol{v}}(1)\right\} .
\end{gathered}
$$

The first product on the right-hand side of (16) converges in the large system to [6], [7]

$$
\frac{1}{N_{t}} \hat{\boldsymbol{v}}^{\dagger}(1) \boldsymbol{H}^{\dagger}(1) \boldsymbol{H}(1) \hat{\boldsymbol{v}}(1) \longrightarrow \gamma_{\mathrm{rvq}}^{\infty}\left(\frac{\bar{B}}{L}\right)
$$

for $\bar{N}_{r}>0$. (We will treat $\bar{N}_{r}=0$ in the next section.) For the second term in (16), the unit-norm $\hat{\boldsymbol{v}}(1)$ is independent of
$\boldsymbol{W}(k-i)$ and hence,

$$
\frac{1}{N_{t}} \hat{\boldsymbol{v}}^{\dagger}(1) \boldsymbol{W}^{\dagger}(k-i) \boldsymbol{W}(k-i) \hat{\boldsymbol{v}}(1) \longrightarrow \bar{N}_{r} .
$$

The third and fourth terms are the cross terms consisting of independent matrices with zero mean and thus, converge to zero.

Therefore, we have that for $\bar{N}_{r}>0$,

$$
\begin{aligned}
\lim _{\left(N_{t}, N_{r}, B\right) \rightarrow \infty} & \frac{1}{N_{t}} \hat{\boldsymbol{v}}^{\dagger}(1) \boldsymbol{H}^{\dagger}(k) \boldsymbol{H}(k) \hat{\boldsymbol{v}}(1) \\
& =\alpha^{2 k-2} \gamma_{\mathrm{rvq}}^{\infty}\left(\frac{\bar{B}}{L}\right)+\bar{N}_{r}\left(1-\alpha^{2}\right) \sum_{i=0}^{k-2} \alpha^{2 i} \\
& =\bar{N}_{r}+\alpha^{2 k-2}\left[\gamma_{\mathrm{rvq}}^{\infty}\left(\frac{\bar{B}}{L}\right)-\bar{N}_{r}\right]
\end{aligned}
$$

Consequently, we obtain the following expression for asymptotic capacity difference

$$
\begin{aligned}
\bar{C}_{\triangle}^{\infty} & =\lim _{\left(N_{t}, N_{r}, B\right) \rightarrow \infty} \bar{C}_{\triangle} \\
& =\frac{L}{M} \sum_{k=1}^{K} \log \left(\bar{N}_{r}+\alpha^{2 k-2}\left[\gamma_{\mathrm{rvq}}^{\infty}\left(\frac{\bar{B}}{L}\right)-\bar{N}_{r}\right]\right) \\
& =\frac{1}{K} \sum_{k=1}^{K} \log \left(\bar{N}_{r}+\alpha^{2 k-2}\left[\gamma_{\mathrm{rvq}}^{\infty}\left(\frac{\bar{B} K}{M}\right)-\bar{N}_{r}\right]\right) .
\end{aligned}
$$

we would like to maximize the asymptotic capacity difference over feedback interval $K$. For a given feedback bits $\bar{B}$ per $M$ blocks and $\bar{N}_{r}>0$, the optimal feedback interval that maximizes the asymptotic capacity difference is given by

$$
K^{*}=\arg \max _{\substack{1 \leq K \leq M \\ K \in \mathbb{Z}^{+}}}\left[\prod_{k=1}^{K} \bar{N}_{r}+\alpha^{2(k-1)}\left[\gamma_{\mathrm{rvq}}^{\infty}\left(\frac{\bar{B} K}{M}\right)-\bar{N}_{r}\right]\right]^{\frac{1}{K}}
$$

where $\mathbb{Z}^{+}$denotes the set of all positive integers.

Finding a closed-form solution for $K^{*}$ in (24) may not be possible in general because of integer optimization. However, a numerical solution is always possible by exhaustive search. We note that the optimal feedback interval in (24) will depend on the temporal correlation coefficient, amount of feedback, and the number of transmit and receiver antennas. Next we consider two extreme regimes for which $\alpha \rightarrow 0$ and $\alpha \rightarrow$ 1 . When the channel does not change $(\alpha \rightarrow 1)$, the optimal feedback interval equals $M$ as follows

$$
\lim _{\alpha \rightarrow 1} K^{*}=M \text {. }
$$

This implies that there must be only one feedback update with all available feedback bits. The analytical result shown seems to agree with intuition. To show (25), we take the limit on both sides of (24) and obtain

$$
\lim _{\alpha \rightarrow 1} K^{*}=\arg \max _{\substack{1 \leq K \leq M \\ K \in \mathbb{Z}^{+}}} \gamma_{\mathrm{rvq}}^{\infty}\left(\frac{\bar{B} K}{M}\right)=M
$$

since $\gamma_{\mathrm{rvq}}^{\infty}(\cdot)$ is an increasing function. 
With some manipulation, we can deduce from (24) that

$$
\lim _{\alpha \rightarrow 0} K^{*}=\arg \max _{\substack{1 \leq K \leq M \\ K \in \mathbb{Z}^{+}}} \frac{1}{K} \log \left(\frac{1}{\bar{N}_{r}} \gamma_{\text {rvq }}^{\infty}\left(\frac{\bar{B} K}{M}\right)\right) .
$$

Similar to (24), the solution to the problem in (27) can be found by exhaustive search. Clearly we see a performance tradeoff over $K$. Small $K$ results in larger number of feedback intervals, but fewer feedback bits per interval. On the other hand, large $K$ leads to smaller number of feedback intervals, but larger number of feedback bits per interval.

\section{Large System With $\bar{N}_{r} \rightarrow 0$}

The main result obtained previously only apply to a large multiantenna system with $\bar{N}_{r}>0$. In this section, we examine the case in which the receiver is equipped with only single antenna (MISO channel) or the case in which the number of receive antennas increases less than linearly with that of transmit antennas. For both cases mentioned, $\bar{N}_{r} \rightarrow 0$ in a large system limit.

First we evaluate the large system limit of $\frac{1}{N_{t}} \hat{\boldsymbol{v}}^{\dagger}(1) \boldsymbol{H}^{\dagger}(k) \boldsymbol{H}(k) \hat{\boldsymbol{v}}(1)$, which consists of four terms as shown in (16). For $\bar{N}_{r}=0$, [6] showed that

$$
\begin{aligned}
\frac{1}{N_{t}} \hat{\boldsymbol{v}}^{\dagger}(1) \boldsymbol{H}^{\dagger}(1) \boldsymbol{H}(1) \hat{\boldsymbol{v}}(1) & \longrightarrow \gamma_{\mathrm{rvq}}^{\infty}\left(\frac{\bar{B} K}{M}\right) \\
& =1-2^{-\frac{\bar{B} K}{M}}
\end{aligned}
$$

while

$$
\frac{1}{N_{t}} \hat{\boldsymbol{v}}^{\dagger}(1) \boldsymbol{W}^{\dagger}(k-i) \boldsymbol{W}(k-i) \hat{\boldsymbol{v}}(1) \longrightarrow 0 .
$$

Thus, the asymptotic capacity difference with $\bar{N}_{r}=0$ is given by

$$
\begin{aligned}
\bar{C}_{\triangle}^{\infty}\left(\bar{N}_{r}=0\right) & =\frac{1}{K} \sum_{k=1}^{K} \log \left(\alpha^{2 k-2}\left(1-2^{-\frac{\bar{B} K}{M}}\right)\right) \\
& =(K-1) \log (\alpha)+\log \left(1-2^{-\frac{\bar{B} K}{M}}\right)
\end{aligned}
$$

for $0<\alpha \leq 1$.

Maximizing the asymptotic capacity difference in (32) gives the optimal feedback interval as follows. For $\bar{N}_{r}=0$ and $0<\alpha \leq 1$, the optimal feedback interval is determined by

$$
K^{*}=\arg \max _{\substack{1 \leq K \leq M \\ K \in \mathbb{Z}^{+}}} \alpha^{K-1}\left(1-2^{-\frac{\bar{B} K}{M}}\right) .
$$

If we remove the integer constraint, we can find the maximum by examining the first derivative of $\bar{C}_{\triangle}^{\infty}\left(\bar{N}_{r}=0\right)$ in (32) and obtain the following approximation

$$
K^{*} \approx \frac{M}{\bar{B}} \log _{2}\left(1+\frac{\bar{B} \log 2}{M \log \frac{1}{\alpha}}\right)
$$

where $0<\alpha<1$. We note that for large available feedback $\bar{B}$, $K^{*}$ is small. The solution implies that feedback update should occur often when a large number of feedback bits is available.
The more practical regime is a small-feedback regime. When $\bar{B} \rightarrow 0$, the optimal $K$ is approximated as follows

$$
\lim _{\bar{B} \rightarrow 0} K^{*} \approx \frac{\log 2}{\log \frac{1}{\alpha}} .
$$

We note that $K^{*}$ is increasing with $\alpha$. Thus, we can conclude that with low feedback rate and highly correlated channel, the feedback interval should be large and the feedback update should occur less frequently.

\section{Numerical Results}

In Fig. 1, we compare the capacity difference of a large system derived in (23) with that of a finite-size system for various $\bar{N}_{r}, \bar{B}$, and $\alpha$. We see that as the system size increases, the simulation results approach the large system results. For a smaller system (e.g., $4 \times 4$ ), the performance gap between numerical simulations and the large system limit could be large. however, both the numerical results and the large system limits display the same trend. In Fig. 1, the feedback interval is fixed at $K=8$. As $\bar{B}$ increases, performance of the channel increases at the different rate for different $\alpha$. When the channel is less correlated $(\alpha=0.5)$, the quantized beamformer of the first block is not a good substitute for that of the next blocks. That is why we do not see much gain although the total feedback is increased.

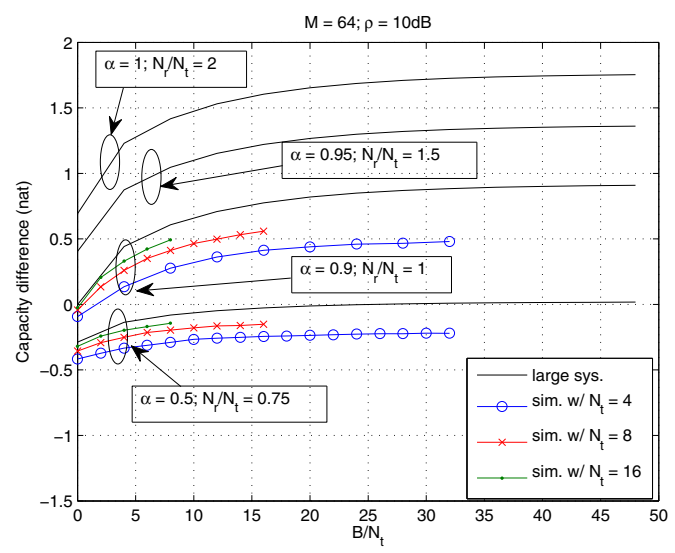

Fig. 1. The large system results are compared with those of finite-size systems with $M=64$, and $\rho=10 \mathrm{~dB}$

Figs. 2 and 3 show the asymptotic capacity difference with feedback interval $K$ for MIMO and MISO channels, respectively. In Fig. 2, selecting the optimal feedback interval $K^{*}=4$ performs about $30 \%$ higher than setting $K=1$, which corresponds to feeding back for every fading block. For channel with low temporal correlation ( $($ mall $\alpha$ ), feedback should take place at every fading block. We also compare the numerical results with the large system ones. Although the difference between the two results can be large for a small MIMO system. The optimal $K^{*}$ 's from the two results are very close.

In Fig. 4, we plot the optimal feedback interval $K^{*}$ found by (24) for MIMO channel and by (33) for MISO channel 


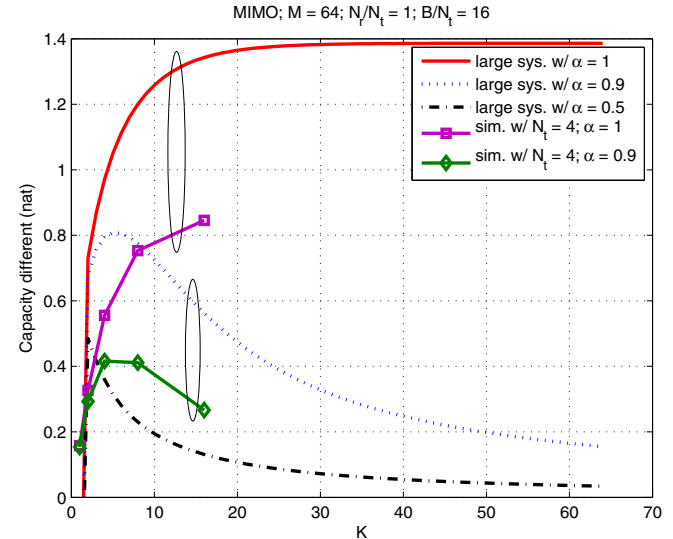

Fig. 2. The large system performance for MIMO channel is shown with $K$ for $M=64, \bar{N}_{r}=1$, and $\bar{B}=16$.

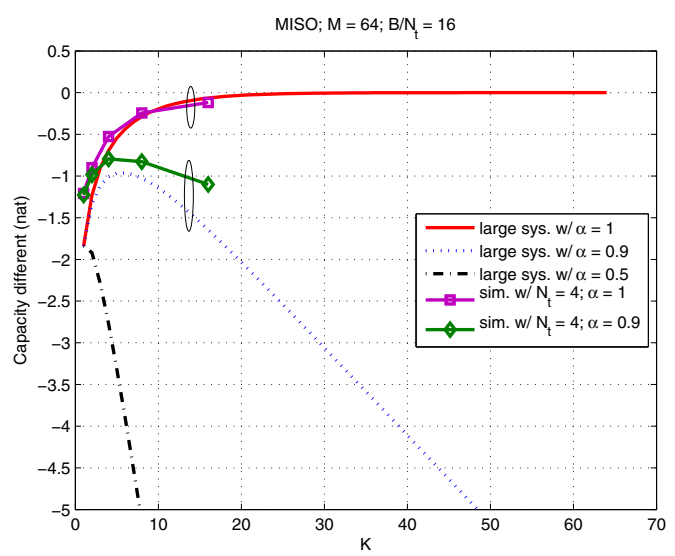

Fig. 3. The large system performance for MISO channel is shown with $K$ for $M=64, \bar{N}_{r}=0$, and $\bar{B}=16$.

versus $\alpha$. We note that $K^{*}$ increases with $\alpha$ as expected. For a low-feedback regime, the optimal feedback interval is greater than the one with small temporal correlation.

\section{CONCLUSiOns}

We have determined the integer optimization problem to find the optimal feedback interval, which can be used as a guideline for a system designer in a practical multiantenna wireless system. In a very limited feedback regime, the optimal feedback interval for MISO channel largely only depends on the temporal correlation and also increases with it. We expect similar results for MIMO channel as well. As the numerical results show, operating at the optimal feedback interval can perform as high as $30 \%$ over feeding back for every fading block.

In the model considered, we are only concerned with flatfading single-user channel. The more practical model may also include multipath fading and/or multiuser channel. For our future work, we will extend the results shown here with transmit beamforming to those with arbitrary-rank transmit precoding.

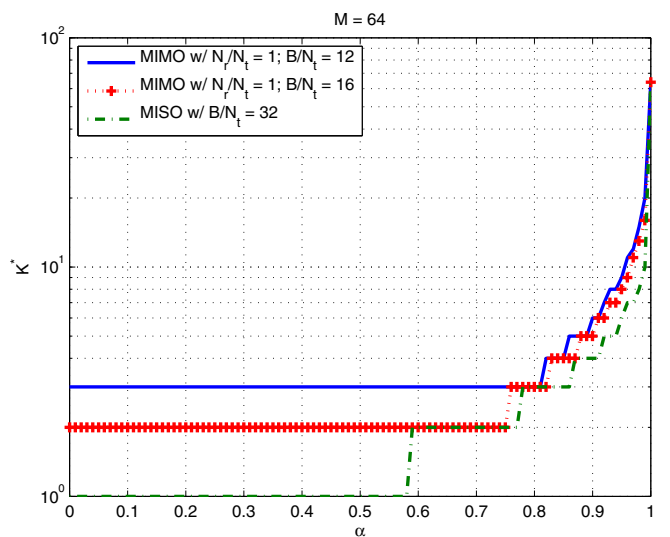

Fig. 4. The optimal feedback interval $K^{*}$ is shown with $\alpha$ for MIMO channel with $\bar{N}_{r}=1$ and MISO channel.

\section{REFERENCES}

[1] I. E. Telatar, "Capacity of multi-antenna Gaussian channels," European Trans. on Telecommun., vol. 10, pp. 585-595, Nov. 1999.

[2] G. J. Foschini and M. J. Gans, "On limits of wireless communications in a fading environment when using multiple antennas," Wireless Personal Commun., vol. 6, no. 3, pp. 311-335, Mar. 1998.

[3] M. Médard, "The effect upon channel capacity in wireless communication of perfect and imperfect knowledge of the channel," IEEE Trans. Inf. Theory, vol. 46, no. 3, pp. 933-946, May 2000.

[4] B. Hassibi and B. M. Hochwald, "How much training is needed in a multiple-antenna wireless link?" IEEE Trans. Inf. Theory, vol. 49, no. 4, pp. 951-964, Apr. 2003.

[5] D. J. Love, R. W. Heath, Jr., V. K. N. Lau, D. Gesbert, B. D. Rao, and M. Andrews, "An overview of limited feedback wireless communication systems," IEEE J. Sel. Areas Commun., vol. 26, no. 8, pp. 1341-1365, Oct. 2008.

[6] W. Santipach and M. L. Honig, "Capacity of a multiple-antenna fading channel with a quantized precoding matrix," IEEE Trans. Inf. Theory, vol. 55, no. 3, pp. 1218-1234, Mar. 2009.

[7] W. Dai, Y. Liu, and B. Rider, "The effect of finite rate feedback on CDMA signature optimization and MIMO beamforming vector selection," IEEE Trans. Inf. Theory, vol. 55, no. 8, pp. 3651-3669, Aug. 2009.

[8] D. J. Love and R. W. Heath, Jr., "Grassmannian beamforming for multiple-input multiple-output wireless systems," IEEE Trans. Inf. Theory, vol. 49, no. 10, pp. 2735-2745, Oct. 2003.

[9] B. Mondal and R. W. Heath, Jr., "Channel adaptive quantization for limited feedback MIMO beamforming systems," IEEE Trans. Signal Process., vol. 54, no. 12, pp. 4717-4729, Dec. 2006.

[10] K. Huang, R. W. Heath, Jr., and J. G. Andrews, "Limited feedback beamforming over temporally-correlated channels," IEEE Trans. Signal Process., vol. 57, no. 5, pp. 1-18, May 2009.

[11] T. Kim, D. J. Love, and B. Clerckx, "A feedback update control scheme for limited feedback multiple antennas systems," in Proc. IEEE Global Commun. Conf. (Globecom), Miami, FL, USA, Dec. 2010, pp. 1-5.

[12] _ - "Leveraging temporal correlation for limited feedback multiple antennas systems," in Proc. IEEE Int. Conf. on Acoustic, Speech and Sig. Proc., Dallas, TX, USA, Mar. 2010, pp. 3422-3425.

[13] D. J. Love, R. W. Heath, Jr., W. Santipach, and M. L. Honig, "What is the value of limited feedback for MIMO channels?" IEEE Commun. Mag., vol. 42, no. 10, pp. 54-59, Oct. 2004.

[14] Y. Zhao, M. Zhao, L. Xiao, and J. Wang, "Capacity of time-varying Rayleigh fading MIMO channels," in Proc. IEEE Int. Symp. on Personal, Indoor and Mobile Radio Commun. (PIMRC), Berlin, Germany, Sep. 2005, pp. 547-551.

[15] C. B. Peel and A. L. Swindlehurst, "Throughput-optimal training for a time-varying multi-antenna channel," IEEE Trans. Wireless Commun., vol. 6, no. 9, pp. 3364-3373, Sep. 2007. 